\title{
THE ANALYSIS OF ELECTRODE IMPEDANCES COMPLICATED BY THE PRESENCE OF A CONSTANT PHASE ELEMENT
}

\author{
G J BRUG, A L G VAN DEN EEDEN, M SLUYTERS-REHBACH and J H SLUYTERS \\ Van ' $t$ Hoff Laboratory of Physical and Collotd Chemistry, State University Utrecht, Padualaan 8, \\ 3584 CH Utrecht (The Netherlands)
}

(Recelved 8th February 1984, in revised form 27th March 1984)

\begin{abstract}
The electrical double-layer at a solid electrode does not in general behave as a pure capacitance but rather as an impedance displaying a frequency-independent phase angle different from $90^{\circ}$ Ways are indicated how to analyse the interfacial impedance if such a complication arises in the presence of a faradaic process, both on the supposition that the double-layer behaviour is due to surface inhomogeneity and on the supposition that it is a double-layer property per se

As examples, the equations derived are successfully applied to a totally irreversible and an ac quasi-reversible electrode process at a gold electrode
\end{abstract}

\section{(I) INTRODUCTION}

According to conventional double-layer theories, the impedance $Z$ of an ideally polarized electrode consists of a capacitance $\left(C_{\mathrm{d}}\right)$ in series with the solution resistance $\left(R_{\Omega}\right)$, and consequently the corresponding complex plane diagram ( $Z^{\prime \prime}$ vs. $Z^{\prime}$ ) should exhibit a straight vertical line intersecting the horizontal axis at $Z^{\prime}=R_{\Omega}$, while the ordinates along this line equal $Z^{\prime \prime}=\left(\omega C_{\mathrm{d}}\right)^{-1}$.

This ideal behaviour is experimentally best observed at mercury/(aqueous) solution interfaces, for example at a dropping mercury electrode (DME), a hanging mercury drop electrode (HMDE), or a mercury pool electrode. Deviations from 1deality may occur in these cases, owing to rather trivial effects such as capillary response or creeping [1], shielding by the glass capillary [2,3], and contamination of the mercury surface. The result of such effects is usually a curved complex plane plot, approaching the straight vertical line at higher frequencies. A less trivial deviation may be caused by diffusion-controlled adsorption of species present at a low concentration.

Also at solid electrodes interfering effects, especially contamination and surface roughness, are likely to be present, but apart from these a more marked behaviour 
has frequently been observed and reported [4-8]: instead of a vertical straight line, the complex impedance diagram exhibits a straight line intersectıng the $Z^{\prime}$-axis at $\left(R_{\Omega}, 0\right)$ at an angle smaller than $90^{\circ}$. In other words, the electrode impedance consists of a resitance $R_{\Omega}$ in series with a complex impedance $Z_{\mathrm{c}}$ with the special property that its phase angle is independent of frequency. This phenomenon of the "constant phase angle", mentioned for the first tıme by Frıcke [9], gives reason to designate $Z_{c}$ by the name "constant phase element" (CPE), as will be done throughout this paper. The CPE is generally believed to originate from a distribution in the current density along the electrode surface as a result of surface inhomogeneity. This can be inferred from the analogy with the behaviour of porous electrodes (and other electrodes with intentional inhomogeneity), which has been extensively discussed by de Levie $[4,10]$.

The presence of the electrical double-layer has to be accounted for thoroughly and precisely in the ac-impedance method for the study of (faradaic) electrode processes. In the well-known Randles's equivalent circuit [11], this is done by considering the interfacial admittance $Y_{\mathrm{E}}$ as being the sum of the faradaic admittance $Y_{\mathrm{F}}=Z_{\mathrm{F}}^{-1}$ and the double-layer admittance $Y_{\mathrm{c}}=Z_{\mathrm{c}}^{-1}$, with $Y_{\mathrm{c}}=\imath \omega C_{\mathrm{d}}$ as usual. The total impedance is given by $Z=R_{\Omega}+Y_{\mathrm{E}}^{-1}$. Evidently this "Randles behaviour" will no longer hold if $Z_{\mathrm{c}}$ and thus $Y_{\mathrm{c}}$ have to represent the properties of the constant phase element.

In the first place it is clear that the frequency spectrum of the measured impedances or admittances will be different if, instead of $Y_{\mathrm{c}}=\imath \omega C_{\mathrm{d}}$, a complex admittance $Y_{\mathrm{CPE}}$ with a concomitant non-linear frequency dependence is introduced. As yet, it is not known how this affects the quality and the amount of information on the faradaic process to be gained, or how the analysis procedures can be modified at best.

In addition, it is questionable whether the faradaic admittance itself can still be described mathematically in the conventional way, or rather should be given a modified mathematical description in the case where the interfacial structure is supposed to be inhomogeneous. This question is difficult to answer a priori as long as the fundamental origin of the inhomogeneous character is unclear.

Thus far, little or no attention has been pald to the above-mentioned problems, even from the experımental point of view. Usually, the CPE is merely accepted as such, thus giving rise to one more unknown parameter to which experimental data have to be fitted. It is the purpose of the present paper to provide a more thorough discussion of the matter from a phenomenological point of view. This means that, leaving the fundamental background unexplained, we will try to analyse mathematically some possible cases of distributed behaviour, and discuss their consequences for procedures for analysing experimental data. Next, as an illustration, this methodological theory will be applied to the study of two electrode reactions, viz. (a) the reduction of $\mathrm{H}^{+}$to $\mathrm{H}_{2}$ and (b) the reduction of the tris-oxalato ferric ion to the tris-oxalato ferrous ion $\left(\mathrm{Fe}(\mathrm{Ox})_{3}^{3-} / \mathrm{Fe}(\mathrm{Ox})_{3}^{4-}\right)$, both at a gold electrode. Finally, some conclusions and implications for the study of other systems will be discussed briefly. 


\section{(II) THEORY}

\section{(II.1) The ideally polarized electrode}

The basic assumption in this section will be that the impedance $Z$ of the ideally polarized electrode behaves in conformity with the complex plane plot represented by the dashed straight line in Fig. 1a throughout the whole frequency range $0<\omega<\infty$, where $\omega$ is the angular frequency $\left(\mathrm{s}^{-1}\right)$. The admittance $Y=Z^{-1}$ is accordingly represented by the dashed circular arc in Fig. 1b, with the typical property of its centre being situated underneath the horizontal axis, as indicated. To put it in another way, the plots corresponding to "ideal" behaviour (drawn lines) have to be rotated clockwise by an angle $\psi=\alpha \pi / 2$ in order to represent the "CPE behaviour".

Most formally this means that the classical representation of the impedance, $Z=R_{\Omega}+\left(i \omega C_{\mathrm{d}}\right)^{-1}$, is replaced by an expression of the form [8]:

$Z=R_{\Omega}+(\iota \omega)^{-(1-\alpha)} Q$

and correspondingly the admittance $Y=Z^{-1}$ is given by

$Y=\frac{1}{R_{\Omega}} \frac{(l \omega)^{1-\alpha} R_{\Omega} Q^{-1}}{1+(l \omega)^{1-\alpha} R_{\Omega} Q^{-1}}$

where $R_{\Omega}$ is the resistive limit of $Z$ at $\omega \rightarrow \infty$, and $Q$ is a constant (with dimensions $\left.\Omega \mathrm{cm}^{2} \mathrm{~s}^{-(1-\alpha)}\right)$ representative for the CPE. The dimensionless parameter $\alpha$, which
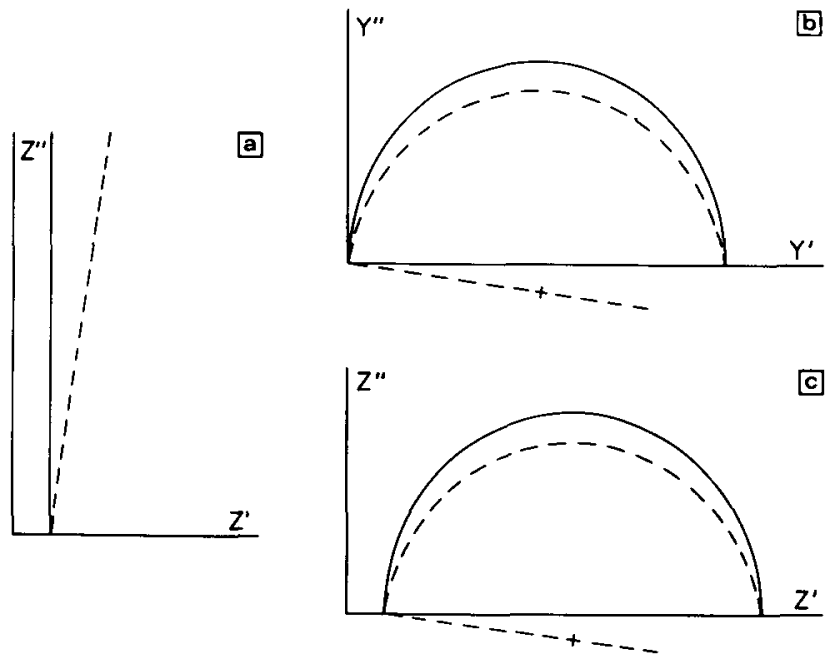

Fig. 1. Impedance and admittance plots of the equivalent circuit of Fig $2 a$ (a and b) and of Fig 4a (c) Dashed curves $\alpha=01$, solid curves $\alpha=0$ 
has a value between zero and unity, is obviously related to the angle of rotation in Fig. 1. Equations (1a) and (1b) are such that the Kramers-Kronig relations $[8,12]$ apply to the real and imaginary components, indicatıng that, in principle, this admittance could have physical relevance:

$Z=Z^{\prime}-\imath Z^{\prime \prime}=R_{\Omega}+\omega^{-(1-\alpha)} Q\{\sin (\alpha \pi / 2)-\imath \cos (\alpha \pi / 2)\}$

and

$Y=Y^{\prime}+\imath Y^{\prime \prime}=\frac{1}{R_{\Omega}} \frac{\omega^{1-\alpha} R_{\Omega} Q^{-1}+\sin (\alpha \pi / 2)+\imath \cos (\alpha \pi / 2)}{\omega^{1-\alpha} R_{\Omega} Q^{-1}+\omega^{-(1-\alpha)} R_{\Omega}^{-1} Q+2 \sin (\alpha \pi / 2)}$

In addition to these expressions, it will be useful for the considerations that follow, to define the "admittance of the CPE" as $Y_{\mathrm{CPE}}=\left(Z-R_{\Omega}\right)^{-1}$ :

$$
Y_{\mathrm{CPE}}=(\iota \omega)^{1-\alpha} Q^{-1}=\omega^{(1-\alpha)} Q^{-1}\{\sin (\alpha \pi / 2)+\imath \cos (\alpha \pi / 2)\}
$$

It should be emphasized that in this way merely the phenomenological behaviour of the solid electrode/electrolyte interface is mathematically described in terms of an equivalent circuit consisting of a resistance $R_{\Omega}$ in series with a CPE (see Fig. 2a). One could think of a more physical meaning of eqns. (1)-(3) by noticing the analogy with the formula developed by Cole and Cole [13] in their treatment of the alternating-current characteristics of dielectric materials where similar rotations of the complex plane loci are frequently observed. To this end, we rewrite eqns. (1a) and (1b) as follows:

$$
\begin{aligned}
& Z=R_{\Omega}\left[1+\left(l \omega \tau_{0}\right)^{-(1-\alpha)}\right] \\
& Y=\frac{1}{R_{\Omega}} \frac{\left(l \omega \tau_{0}\right)^{1-\alpha}}{1+\left(l \omega \tau_{0}\right)^{1-\alpha}}=\frac{1}{R_{\Omega}}\left[1-\frac{1}{1+\left(l \omega \tau_{0}\right)^{1-\alpha}}\right]
\end{aligned}
$$

where $\tau_{0}=R_{\Omega} C_{0}$ may be called the relaxation tıme, evidently related to the existence of the electrical double-layer. Comparison of eqns. (1a) and (4a) leads to

$Q^{-1}=R_{\Omega}^{-1} \tau_{0}^{1-\alpha}=C_{0}^{1-\alpha} R_{\Omega}^{-\alpha}$

回

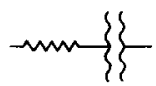

b

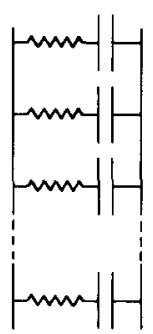

Fig 2 Cell impedance analogues for an ideally polarized electrode (a) Resistance in series with a CPE, (b) ladder network 
if it is postulated that in our case the observed behaviour is due to inhomogeneity of the electrode surface in such a way that only the value of the double-layer capacity is distributed along the interface. As a consequence, the equivalent circuit of the electrode in contact with the homogeneous electrolyte phase should be represented by a ladder network of $R_{\Omega} C$ elements as shown in Fig $2 \mathrm{~b}$. The admittance of this network becomes

$Y=\frac{1}{R_{\Omega}} \int_{0}^{\infty} \frac{\imath \omega \tau}{1+i \omega \tau} G(\tau) \mathrm{d} \tau=\frac{1}{R_{\Omega}}\left[1-\int_{0}^{\infty} \frac{1}{1+\imath \omega \tau} G(\tau) \mathrm{d} \tau\right]$

where $\tau=R C$ and $G(\tau)$ is the distribution function of $\tau$. Fuoss and Kirkwood [14] have derived a procedure to find the distribution function necessary to account for a certain phenomenological behaviour of a complex quantity like $Y$. As this procedure proves to be applicable to our problem, the general result can be applied as well. This means that the following relations hold:

$\tau G(\tau)=F(s) \quad$ with $s=\ln \left(\tau / \tau_{0}\right)$

$\pi F(s)=H(s+\imath \pi / 2)+H(s-\imath \pi / 2)$

where $H(x)$ is the empirical or phenomenological function that describes the dependence of the imaginary component $Y^{\prime \prime}$ on $x=\ln \left(1 / \omega \tau_{0}\right)$, i.e. in our case:

$H(x)=\frac{1}{R_{\Omega}} \frac{\cos (\alpha \pi / 2)}{\exp [(1-\alpha) x]+\exp [-(1-\alpha) x]+2 \sin (\alpha \pi / 2)}$

as follows from eqn. (2b) with $R_{\Omega} Q^{-1}=\tau_{0}^{1-\alpha}$. From eqns. (8) and (9) we find

$F(s)=\frac{1}{2 \pi} \frac{2 \sin (\alpha \pi)}{\exp [(1-\alpha) s]+\exp [-(1-\alpha) s]-2 \cos (\alpha \pi)}$

It is useful to consider some mathematical properties of this function. In Fig. 3,

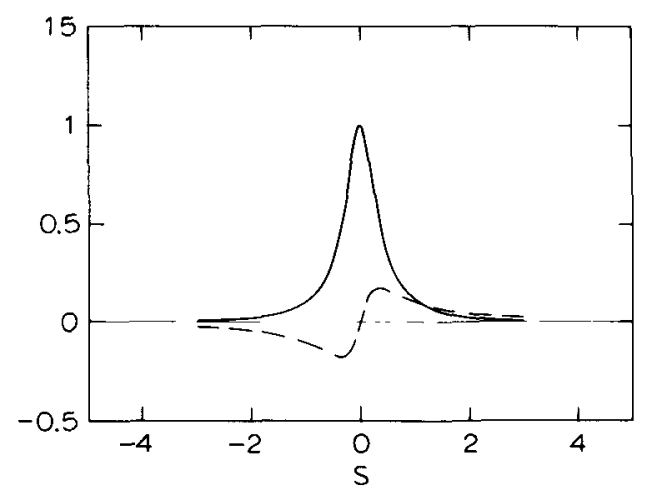

Fig 3 Distribution functions according to eqn (10). Solıd curve $F(s)$, dashed curve. $s F(s), \alpha=01$ 
$F(s)$ and $s F(s)$ are graphically represented as functions of $s$. The integrals of these graphs are

$\int_{-\infty}^{\infty} F(s) \mathrm{d} s=\int_{0}^{\infty} G(\tau) \mathrm{d} \tau=1$

and

$\int_{-\infty}^{\infty} s F(s) \mathrm{d} s=0$

So, the mean value of $s=\ln \left(\tau / \tau_{0}\right)$ equals zero, and thus the mean of $\ln \tau$ is given by

$\langle\ln \tau\rangle=\ln \tau_{0}$

However, the integral $\int_{0}^{\infty} \tau G(\tau) \mathrm{d} \tau \equiv\langle\tau\rangle$ becomes infinite unless $\alpha$ equals zero (i.e. the "ideal" behaviour). This is inherent to the symmetry of the logarithmic distribution function in eqn. (10), which was invoked to account for the phenomenological frequency dependence of the electrode admittance. It must therefore be concluded that eqn. (10) provides merely a mathematical description of experimental facts as well as eqns. (1)-(3). A similar conclusion was reached by Cole and Cole in their treatment of the behaviour of dielectrics [13].

The necessity of such an inconcervable distribution function was ruled out in a sophistıcated treatment by Scheider [7], postulatıng that a so-called branched ladder RC network rather than a single RC ladder should be used to represent the model accounting for the CPE. It is shown that the value of $\alpha$ is determined by the order of branching, irrespective of the distribution in the elements. The physical explanation is thought to be fundamentally related to microscopical surface roughness, causing uneven charging of the double-layer and hence tangential displacements of charge in the diffuse double-layer. The most important motive for this model is the empirical evidence that the apparent double-layer capacity $C_{\mathrm{P}}=Y_{\mathrm{CPE}}^{\prime \prime} / \omega$ is proportional to $R_{\Omega}^{-\alpha}$ [7]. However, in our opinion, the experiments performed by Scheider to prove this relation should be considered with some care, as the data were measured at polarizable gold electrodes without fixing the dc potential.

In view of the discussions to follow, we wish to point out another possible physical background of the CPE, viz. the idea that it is just an intrinsic property of the electrical double-layer, the atoms, molecules and 1ons in it being subject to extremely high electric field strengths and fluctuations therein. It is conceivable that the resulting atomic movements in the interfacial structure, occurring on application of the ac potential, are accompanied by a dissipation of energy during a cycle of the ac perturbation as a consequence of mechanical coupling to neighbourıng atoms. As Cole and Cole have pointed out $[13,15]$, the special property of this process should be the frequency independence of the ratio of maximum energy stored to the energy dissipated per cycle. In this concept there would be no reason for the solution resistance to influence the CPE and consequently the parameter $Q$ in eqns. (1)-(3) is a property of the electrical double-layer only, i.e. $Q^{-1} \neq \mathrm{f}\left(R_{\Omega}\right)$, contrary to the case of the distribution model, see eqn. (5). 


\section{(II 2) Charge density and capacitance of the ideally polarized electrode}

Apart from the question which model is more probable, the CPE behaviour, if observed, causes a problem with respect to the study of the double-layer. Normally, capacity measurements are frequently employed to determine double-layer properthes such as charge density, adsorption, etc. as a function of the dc potential. However, if CPE behaviour is observed, i.e. if $\alpha \neq 0$, it is by no means clear what value should be assigned to the thermodynamic quantity $C_{\mathrm{d}}=\mathrm{d} q_{\mathrm{M}} / \mathrm{d} E$, where $q_{\mathrm{M}}$ is the charge density on the electrode.

Attempts to determine $q_{\mathrm{M}}$ directly, e.g. from potentiostatic pulse or step measurements, can be expected to fail as well. From eqn. (3) it appears that the CPE admittance contains a resistive component parallel to the capacitive component, so that on application of a (constant) potential perturbation from a certain initial state, charge will pass the interface perpetually. A similar argument can be deduced from the potential response to a constant current perturbation, which has been shown to be proportional to $t^{1-\alpha}$ [8], whereas the charge consumed is proportional to $t$.

Also in this respect it has to be concluded that the present state of knowledge about the physical properties of the solid metal solution interface is as yet unsatisfactory. In any case, it is clear that "double-layer capacities" determined by $C_{\mathrm{d}}=Y_{\mathrm{E}}^{\prime \prime} / \omega$, as is frequently done in the literature [16], will be found to depend on the applied frequency, and are therefore of limited significance. At present, it seems more appropriate to select $C_{0}$ as the quantity representing the "double-layer capac1tance", to be obtained as $C_{0}=\left(Q^{-1} R_{\Omega}^{\alpha}\right)^{1 / 1-\alpha)}$ (eqn. 5) if the distribution model is adopted.

\section{II.3 The non-ideally polarized electrode}

\section{(II.3.1) Irreversible uniform charge transfer}

At first, it will be supposed that a simple electrode reaction $\mathrm{O}+n e^{-} \rightleftarrows \mathrm{R}$ is proceeding, its rate being controlled by slow charge transfer, so that diffusion or any other kind of mass transfer need not be accounted for. Then, in the classical case, a transfer resistance $R_{\mathrm{ct}}$ appears in the Randles's equivalent circuit parallel to the double-layer capacitance $C_{\mathrm{d}}$.

Thus far in the literature, one intuitıvely replaced $C_{\mathrm{d}}$ by the CPE in cases where the CPE behaviour was observed in the absence of the faradaic process, or more generally in cases where a fit assuming normal behaviour of the double-layer proved to be impossible. In other words, impedance or admittance analyses are carried out adopting the equivalent circuit shown in Fig. 4a. Regarding the discussions in the foregoing section, it is obvious that this makes sense only if the CPE is considered to be the intrinsic equivalent of the double-layer admittance, i.e. if eqn. (3) has a fundamental meaning with $Q^{-1} \neq \mathrm{f}\left(R_{\Omega}\right)$. Then the total admittance is most convenently expressed by

$Y=\frac{1}{R_{\Omega}+Y_{\mathrm{E}}^{-1}}=\frac{1}{R_{\Omega}}\left[1-\frac{1}{1+R_{\Omega} Y_{\mathrm{E}}}\right]$ 
where $Y_{E}$ is the interfacial admittance and thus, in view of Fig. 4a and eqn. (3),

$Y=\frac{1}{R_{\Omega}}\left[1-\frac{1}{1+R_{\Omega} R_{\mathrm{ct}}^{-1}+R_{\Omega} Q^{-1}(\iota \omega)^{1-\alpha}}\right]$

However, if the distribution concept is the fundamental background of the CPE behaviour, the situation is different. For in that case, the charge transfer proceeding at any location on the surface is shunted by the capacitance pertaining to this location, and therefore the appropriate equivalent cırcuit is a ladder network as drawn in Fig. 4b (or a more complex branched network, if a more sophisticated model is required). The corresponding admittance has to be computed with

$Y=\frac{1}{R_{\Omega}}\left[1-\int_{-\infty}^{\infty} \frac{1}{1+R_{\Omega} R_{\mathrm{ct}}^{-1}+\imath \omega \tau} F(s) \mathrm{d} s\right]$

where $F(s)$ is the same distribution function as before, i.e. given by eqn. (8), and $s=\ln \left(\tau / \tau_{0}\right)$. After introduction of a new parameter $\tau_{0}^{\prime}$ :

$\tau_{0}^{\prime}=\tau_{0} /\left[1+R_{\Omega} R_{\mathrm{ct}}^{-1}\right]$

the integral in eqn. (16) can be rewritten as

$I=\frac{1}{1+R_{\Omega} R_{\mathrm{ct}}^{-1}} \int_{-\infty}^{\infty} \frac{1}{1+\mathrm{i} \omega \tau_{0}^{\prime} \exp (s)} F(s) \mathrm{d} s$

Here the integrand is of the same mathematical form as in the absence of a faradaic process (i.e. $R_{\mathrm{ct}}^{-1}=0$ and $\tau_{0}^{\prime}=\tau_{0}$ ). Therefore it can safely be concluded that the solution of eqn. (18a) is

$I \equiv \frac{1}{1+R_{\Omega} R_{\mathrm{ct}}^{-1}} \frac{1}{1+\left(\iota \omega \tau_{0}^{\prime}\right)^{1-\alpha}}$

回

回
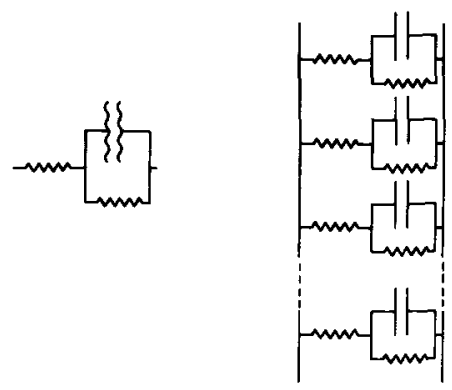

Fig. 4. Cell impedance analogues for an irreversible reaction. (a) Resistance in connection with a CPE, shunted by a charge-transfer resistance; (b) ladder network 
Hence we obtain from eqn. (16):

$Y=\frac{1}{R_{\Omega}}\left[1-\frac{1}{1+R_{\Omega} R_{\mathrm{ct}}^{-1}+\left(\iota \omega \tau_{0}\right)^{1-\alpha}\left(1+R_{\Omega} R_{\mathrm{ct}}^{-1}\right)^{\alpha}}\right]$

where $\tau_{0}$ still equals $R_{\Omega} C_{0}$. The conclusion is that $Q^{-1}$ in eqn. (15) is not replaced by $C_{0}^{1-\alpha} R_{\Omega}^{-\alpha}$ as is usually thought, but by

$Q^{-1}=C_{0}^{1-\alpha}\left[R_{\Omega}^{-1}+R_{\mathrm{ct}}^{-1}\right]^{\alpha}$

Although eqn. (19), as it is deduced from a very simple (or rough) model, may be too simple an approximation, it clearly shows in conjunction with eqns. (15) and (20) that the CPE is coupled not only to the solution resistance but also to the charge-transfer resistance (or more generally to the faradaic impedance), if it is caused by microscopical or sub-microscopical heterogeneity of the electrode surface. To our knowledge, this has not been recognized before.

\section{(II.3.2.) Irreversible distributed charge transfer}

Next, the question arises whether it is philosophically correct to assume the rate of charge transfer to be uniform along the interface, whereas it is assumed that the most characteristic property of the interface, the potential dependence of the charge density, is distributed. Again, the lack of basic understanding prevents a fundamental discussion and even a decision whether the answer to this question is yes or no. Yet it seems useful to consider from the phenomenological point of view what can be expected in the case where the value of $R_{\mathrm{ct}}$ is distributed. Two possibilities will be envisaged, namely the case where $R_{\mathrm{ct}}$ and $C_{\mathrm{d}}$ are distributed independently and the case where the distributions are coupled.

If the distributions are independent, the overall admittance must be of the form

$$
Y=\frac{1}{R_{\Omega}}\left[1-\int_{-\infty}^{\infty} \int_{-\infty}^{\infty} \frac{1}{1+\rho+\imath \omega \tau} G(r) \mathrm{d} r F(s) \mathrm{d} s\right]
$$

with $\rho=\mathrm{R}_{\Omega} \mathrm{R}_{\mathrm{ct}}^{-1}, r=\ln \left(\rho / \rho_{0}\right)$ and $s=\ln \left(\tau / \tau_{0}\right)$. The distribution of $s$ may be supposed to be given by eqn. (10). The feasibility of solving the double integral naturally depends on the nature of $G(r)$, i.e. the distribution of $r$. However, for sufficiently small values of $\rho$, a simplification can be derived by writing:

$\frac{1}{1+\rho+\imath \omega \tau}=\frac{1}{1+\imath \omega \tau}\left[1-\frac{\rho}{1+\imath \omega \tau}+\frac{\rho^{2}}{(1+\imath \omega \tau)^{2}}-\ldots\right]$

The integral over $G(r) \mathrm{d} r$ yields a summation identical to eqn. (22), with $\rho$ and $\rho^{2}$ etc., replaced by their mean value indicated as $\langle\rho\rangle,\left\langle\rho^{2}\right\rangle$, etc. Limiting eqn. (22) to first-order approximation means that eqn. (21) is replaced by

$$
Y=\frac{1}{R_{\Omega}}\left[1-\int_{-\infty}^{\infty} \frac{1}{1+\langle\rho\rangle+\imath \omega \tau} F(s) \mathrm{d} s\right]
$$

irrespective of the nature of the distribution in $\rho$. Since eqn. (23) is similar to eqn. 
(16), it follows that in eqns. (17)-(20) $R_{\mathrm{ct}}^{-1}$ can be replaced by its mean, $\left\langle R_{\mathrm{ct}}^{-1}\right\rangle$. The validity of this result is mainly determined by the condition

$$
\frac{\left\langle\rho^{2}\right\rangle-\langle\rho\rangle^{2}}{(1+\imath \omega \tau)^{3}} \ll \frac{1}{1+\langle\rho\rangle+\imath \omega \tau}
$$

Evidently this condition is favoured if $\left\langle\rho^{2}\right\rangle-\langle\rho\rangle^{2}$, 1.e. the square of the standard deviation in $\rho$, is small, in other words if the distribution of $R_{\mathrm{ct}}^{-1}$ is rather narrow.

In a representative case it can be calculated that the approximation expressed by eqn. (23) holds within $2 \%$ if it is supposed that the standard deviation in $\rho$ is of the same order of magnitude as its mean value $\langle\rho\rangle$.

If 1 is supposed that both the rate of charge transfer and the double-layer capacity are distributed due to a distribution in the state of the surface, it is logical to assume that both $\rho=R_{\Omega} R_{\mathrm{ct}}^{-1}$ and $\tau=R_{\Omega} C$ are functions of the same distribution parameter $s$. Consequently, we have

$Y=\frac{1}{R_{\Omega}}\left[1-\int_{-\infty}^{\infty} \frac{1}{1+\rho(s)+\imath \omega \tau(s)} F(s) \mathrm{d} s\right]$

Naturally the mathematical form of $\rho(s)$ has to be deduced from experimental facts. By analogy to $\tau(s)=\tau_{0} \exp (s)$, one could speculate that $\rho(s)=\rho_{0} \exp (s)$. In that case, the phenomenological behaviour will correspond to

$$
Y=\frac{1}{R_{\Omega}}\left[1-\frac{1}{1+\left(\rho_{0}+\imath \omega \tau_{0}\right)^{1-\alpha}}\right]
$$

It may be worthwhile to compare experimental facts both with eqn. (19) and eqn. (25b), and to analyse whether a distinction can be made. However, a distribution function for $R_{\mathrm{ct}}^{-1}$ of the form of eqn. (10) would, of course, be as unrealistic as that for the double-layer charging process: it means that the average rate of charge transfer becomes infınite.

\section{(II.3.3) Non-trreversible charge transfer}

If in addition to charge transfer the electrode reaction is also controlled by (diffusional) mass transport, this has to be accounted for by introducing the complex faradaic admittance $Y_{\mathrm{F}}$ instead of $R_{\mathrm{ct}}^{-1}$. Algebraic manipulations as in the foregoing sections will be more tedious, but it can be inferred that the conclusions and considerations will be similar and analogous. Therefore we will not treat this case in detail, but only suggest that in eqn. (15)-(24) $R_{\mathrm{ct}}^{-1}$ can be replaced by $Y_{\mathrm{F}}$ if necessary.

It has to be noted that now the frequency spectrum of the overall admittance is governed by the nature of the CPE. For, replacing $R_{\mathrm{ct}}^{-1}$ by $Y_{\mathrm{F}}$ in eqns. (19) and (20) introduces an extra frequency dependence incorporated in $Q^{-1}$, whereas this will not occur in the more simple eqn. (15). In other words, if the distribution model makes sense, this causes a more complex frequency dependence of the term due to the distributed capacity. 


\section{(III.1) Potential dependence}

In the context of our treatment it will be useful to combine experimental results from $\mathrm{dc}$ and ac measurements. To this end, we formulate the following premises:

(i) The rate expression for the electrode reaction $\mathrm{O}+n e^{-} \rightleftarrows \mathrm{R}$ obeys linear stoichiometry; the faradaic current is related to the interfacial potential $E$ by

$J_{\mathrm{F}}=-n F k_{\mathrm{f}}\left[c_{\mathrm{O}}-c_{\mathrm{R}} \exp (\phi)\right]$

where $\mathbf{k}_{\mathrm{f}}$ is the potential-dependent forward rate constant, $c_{\mathrm{O}}$ and $c_{\mathrm{R}}$ are the interfacial concentrations and $\phi=(n F / R T)\left(E-E^{\circ}\right), E^{\circ}$ being the formal standard potential.

(ii) Mass transport occurs by means of semi-infunite linear diffusion and consequently [17] for the potential step method, when $R$ is initially absent,

$J_{\mathrm{F}}=-n F k_{\mathrm{f}} c_{\mathrm{O}}^{*} \exp \left(\lambda^{2} t\right) \operatorname{erfc}\left(\lambda t^{1 / 2}\right)$

with

$\lambda=k_{\mathrm{f}}\left[D_{\mathrm{O}}^{-1 / 2}+D_{\mathrm{R}}^{-1 / 2} \exp (\phi)\right]$

and $c_{\mathrm{O}}^{*}$ is the bulk concentration of $O$.

(iii) Complicating processes like adsorption or non-statıonary multi-step mechanisms [18] are supposed to be absent or of minor influence. The faradaic admittance is therefore given by [18]:

$$
Y_{\mathrm{F}}=Y_{\mathrm{F}}^{\prime}+Y_{\mathrm{F}}^{\prime \prime}=R_{\mathrm{ct}}^{-1} \frac{p^{2}+p+\imath p}{p^{2}+2 p+2}
$$

where $p=(2 \omega)^{1 / 2} / \lambda$ and $R_{\mathrm{ct}}$ is the charge-transfer resistance, obeying the expression

$R_{\mathrm{ct}}^{-1}=\left(\frac{\partial J_{\mathrm{F}}}{\partial E}\right)_{c_{\mathrm{O}}, c_{\mathrm{R}}}=\frac{n F}{R T}\left[J_{\mathrm{F}} \frac{\mathrm{d} \ln k_{\mathrm{f}}}{\mathrm{d} \phi}+\frac{\left(J_{\mathrm{F}}+n F k_{\mathrm{f}} c_{\mathrm{O}}^{*}\right)}{1+\exp (-\phi)}\right]$

For dc irreversible reactions, the second term in eqn. (30) is negligible because of the large value of $\exp (-\phi)$ in the faradaic region. In that case, we have

$\int_{E_{1}}^{E} R_{\mathrm{ct}}^{-1} \mathrm{~d} E=\int_{0}^{k_{\mathrm{f}}(E)} k_{\mathrm{f}}^{-1} J_{\mathrm{F}} \mathrm{d} k_{\mathrm{f}}$

where $E$ is the potential of interest and $E_{1}$ is some initial potential outside the faradaic region, i.e. where $R_{\mathrm{ct}}^{-1}=0$. With the aid of eqn. (27), the integral of the right-hand side of eqn. (31) can be computed as a function of the theoretical $k_{\mathrm{f}}(E)$ values. The left-hand side can be determined from $R_{\mathrm{ct}}^{-1}$ vs. $E$ data and thus enalsles us to find $k_{\mathrm{f}}(E)$. If desired, the results can be checked by comparison with an analysis of the dc current $J_{\mathrm{F}}$ according to eqn. (27).

For dc reversible reactions, both terms containing $J_{F}$ on the right-hand sivie of eqn. (30) vanish and so $k_{\mathrm{f}}(E)$ can be obtained directly from $R_{\mathrm{ct}}^{-1}$. 
If a reaction is quasi-irreversible, a more involved fittıng procedure on $k_{\mathrm{f}}(E)$ is required.

\section{(III.2) Frequency dependence; ac trreversible case}

First it is of some importance to consider the graphical representation of impedance data in the complex impedance plane. If eqn. (15) or (19) is supposed to hold, it is easily derived that on varying the frequency (at fixed potential) the well-known circular arc, as first described by Sluyters [19], will be obtained, but its centre is shifted vertically underneath the horizontal axis (see Fig. 1c). The circle intersects the horizontal axis at the points $R_{\Omega}, 0$ (infinite frequency) and $R_{\Omega}+R_{\mathrm{ct}}, 0$ (zero frequency). The equation of the circle is

$$
\left[Z^{\prime}-R_{\Omega}-\frac{1}{2} R_{\mathrm{ct}}\right]^{2}+\left[Z^{\prime \prime}+\frac{1}{2} R_{\mathrm{ct}} \operatorname{tg}(\alpha \pi / 2)\right]^{2}=\frac{1}{4} R_{\mathrm{ct}}^{2} / \cos ^{2}(\alpha \pi / 2)
$$

and it is interesting to note that the frequency pertaining to the "top" of the circle, where $Z^{\prime \prime}$ is maximum, corresponds to the relation

$\omega_{\mathrm{top}}^{1-\alpha}=Q R_{\mathrm{ct}}^{-1} \quad$ or $\quad \omega_{\mathrm{top}}^{-1}=C_{0} R_{\mathrm{ct}}\left[R_{\mathrm{ct}} / R_{\Omega}+1\right]^{\alpha / 1-\alpha}$

which evidently replaces the "classical" relatıonship $\omega_{\text {top }}=\left(C_{\mathrm{d}} R_{\mathrm{ct}}\right)^{-1}[19]$.

As usual, the evaluation of the system parameters in the whole frequency region will start by calculating the "interfacial admittance" $Y_{\mathrm{E}}$ by means of eqn. (14), where for the ohmic resistance $R_{\Omega}$ a proper value is substituted, e.g. determined by extrapolation of measured data to infinite frequency, preferably at a potential outside the faradaic region. From eqns. (14) and (15), it follows that both the real and the imaginary components of $Y_{\mathrm{E}}$ are functions of $\omega$ following

$Y_{\mathrm{E}}^{\prime}+\imath Y_{\mathrm{E}}^{\prime \prime}=R_{\mathrm{ct}}^{-1}+\omega^{1-\alpha} Q^{-1}[\sin (\alpha \pi / 2)+\imath \cos (\alpha \pi / 2)]$

Consequently a plot of $Y_{\mathrm{E}}^{\prime}$ vs. $Y_{\mathrm{E}}^{\prime \prime}$ at varied frequency should give a straight line according to

$Y_{\mathrm{E}}^{\prime}=R_{\mathrm{ct}}^{-1}+Y_{\mathrm{E}}^{\prime \prime} \operatorname{tg}(\alpha \pi / 2)$

In addition, the validity of eqn. (34) can be examined by verifying that the relation

$\log Y_{\mathrm{E}}^{\prime \prime}=(1-\alpha) \log \omega+\log \left[Q^{-1} \cos (\alpha \pi / 2)\right]$

applies.

Note that the whole analysis is rigorous, irrespective of the origin of the CPE. However, the meaning of the experimentally determined $Q^{-1}$ is not unambiguous.

\section{(III.3) Frequency dependence; general case}

If a reaction proceeds by relatively fast charge transfer, the influence of $p=p^{\prime} \omega^{1 / 2}$ in eqn. (29) becomes significant. As has been argued in Section (II.3.3), to what extent this influences the eventual frequency dependence of $Y_{\mathrm{E}}$ now depends on the origin of the CPE. However, if $\alpha$ is small and/or $\left|Y_{F}\right| \ll R_{\Omega}^{-1}$, eqn. (14) may hold 
within experimental error, in combination with

$Y_{\mathrm{E}}^{\prime}+\imath Y_{\mathrm{E}}^{\prime \prime}=R_{\mathrm{ct}}^{-1} \frac{p^{2}+p+\imath p}{p^{2}+2 p+2}+\omega^{1-\alpha} Q^{-1}[\sin (\alpha \pi / 2)+\imath \cos (\alpha \pi / 2)]$

The analysis of $Y_{\mathrm{E}}$ data, calculated as before from $Y$ and $R_{\Omega}$, requires numerical procedures rather than graphical ones. If $Q^{-1}$ is eliminated between $Y_{\mathrm{E}}^{\prime}$ and $Y_{\mathrm{E}}^{\prime \prime}$, the following relationship results:

$$
Y_{\mathrm{E}}^{\prime}-Y_{\mathrm{E}}^{\prime \prime} \operatorname{tg}(\alpha \pi / 2)=R_{\mathrm{ct}}^{-1} \frac{p^{2}+p[1-\operatorname{tg}(\alpha \pi / 2)]}{p^{2}+2 p+2}
$$

From the frequency dependence of this quantity, the two parameters $p^{\prime}=R_{\mathrm{ct}} / \sigma$ and $R_{\text {ct }}$ can be obtained by a fitting procedure, provided that the value of $\alpha$ is correctly adjusted. it will be favourable if $\alpha$ is known from independent measurements, e.g. outside the faradaic region, or performed in the absence of the electroactive species. If the fit is successful, this may be considered as an argument supporting the validity of the model underlying eqn. (37).

In Fig. 5, the frequency-dependent factor in eqn. (38) has been plotted vs. $\omega^{-1 / 2}$. It should not be ignored that in practice the quantity $Y_{\mathrm{E}}^{\prime}-Y_{\mathrm{E}}^{\prime \prime} \operatorname{tg}(\alpha \pi / 2)$ is obtained after assigning the values of two system parameters, viz. $R_{\Omega}$ and $\alpha$, both being of major influence at high frequencies. Comparison with the theoretical behaviour may give reason to a re-adjustment of these parameters in order to obtain a better fit.

(IV) EXPERIMENTAL

\section{(IV.1) Reduction of protons}

This reaction was studied using aqueous solutions of $1 M \mathrm{HClO}_{4}$, and $1 \mathrm{M}$ $\mathrm{NaClO}_{4}+6.36 \times 10^{-3} \mathrm{M} \mathrm{HClO}_{4}$. Reagents and the preparation of solutions were

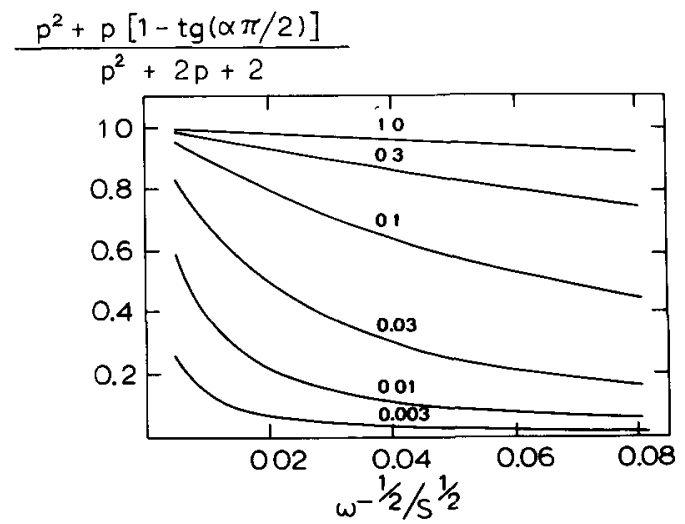

Fig 5. The function $\left[p^{2}+p(1-\operatorname{tg}(\alpha \pi / 2))\right] /\left(p^{2}+2 p+2\right)$ as a function of $\omega^{-1 / 2}$ (see eqn 38), for the indicated values of $p^{\prime}$, taking $\alpha=0.1$ 
the same as those described in ref. 20. Also the electrodes-monocrystalline and polycrystalline gold-were the same as those used before. The electrodes were contacted to the solution by the pendant meniscus method. Reproducibility and stability of the surface state were frequently checked by cyclic voltammetry.

For the dc and ac measurements, the potential step procedure with intermittent sweeps into the oxygen region was applied [20]. Impedance and dc current data were sampled at $t=4 \mathrm{~s}$ after application of the potential step. All potentials are referred to the saturated sodium chloride calomel electrode (SSCE). The cell temperature was always $25^{\circ} \mathrm{C}$.

\section{(IV.2) Reduction of tris-oxalato ferric tons}

This reaction was studied at the polycrystalline gold $(5 \mathrm{~N})$ electrode in contact with a solution containing $1 M \mathrm{~K}_{2} \mathrm{C}_{2} \mathrm{O}_{4}+0.05 M \mathrm{H}_{2} \mathrm{C}_{2} \mathrm{O}_{4}$ and $2 \times 10^{-3} \mathrm{M}$ $\mathrm{Fe}_{2}\left(\mathrm{SO}_{4}\right)_{3}$. The solutions were prepared by dissolving oxalic acid and potassium carbonate (both Merck suprapur) in freshly twice-distilled water. The solutions were deaerated by means of argon.

In oxalate solutions it is not possible to clean the electrode surface electrochemically by sweeping into the oxygen region, since then the gold dissolves to a considerable extent. Therefore the potential was swept only to $+0.25 \mathrm{~V}$ vs. SCE and back to the initial potential $E_{1}=0 \mathrm{~V}$ vs. SCE (saturated calomel electrode), which was found to suffice in this medium. For the actual measurements, the potential was stepped to final potentials ranging from -0.100 to $-0.500 \mathrm{~V}$ vs. SCE. The frequency in the impedance measurements was varied between $80 \mathrm{~Hz}$ and $10 \mathrm{kHz}$ (twelve frequencies).

The cell construction was the same as that for the proton reduction.

\section{(V) RESULTS AND DISCUSSION}

\section{(V.1) The proton reduction from perchloric acıd}

In Fig. 6a, a group of complex impedance plots is represented from the data obtained with the solution $1 M \mathrm{NaClO}_{4}+6.36 \times 10^{-3} \mathrm{M} \mathrm{HClO}_{4}$. The curves are circular arcs with their centres somewhat below the horizontal axis. On extrapolation to infinite frequency, the value of the ohmic resistance is found to be $R_{\Omega}=2.3 \Omega$ $\mathrm{cm}^{2}$. The corresponding $Y_{\mathrm{E}}^{\prime \prime}$ vs. $Y_{\mathrm{E}}^{\prime}$ diagrams are shown in Fig. 6b. Quite good straight lines are obtained with slopes significantly deviating from $90^{\circ}$ (note that the $Y_{\mathrm{E}}^{\prime}$-axis is enlarged by a factor of 10 in order to present the results more clearly).

It is remarkable that the constant phase angles observed at one electrode are not the same at different potentials, but tend to decrease going to more negative potentials (or farther into the faradaic region??). The values of $\alpha$ are collected in Table 1. It appears that at the polycrystalline electrode $\alpha$ is slightly higher than at the single crystal electrodes. This may support the distribution concept since it can be expected that a polycrystalline surface is more heterogeneous. Some experiments 

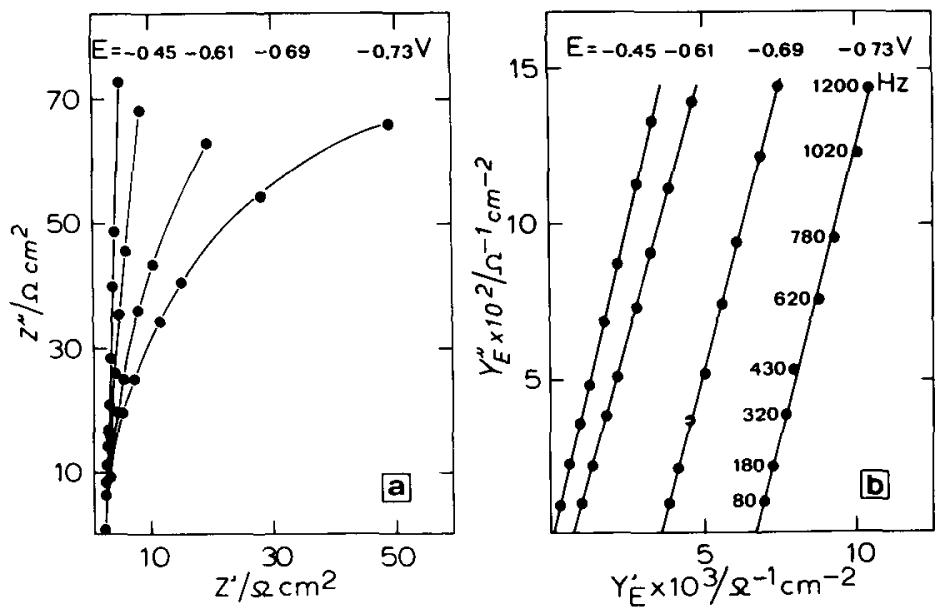

Fig 6 (a) $Z^{\prime \prime}$ vs $Z^{\prime}$ for $\mathrm{H}^{+}$reduction in $6.36 \times 10^{-3} M \mathrm{HClO}_{4}+1 M \mathrm{NaClO}_{4}$ at a polycrystalline gold electrode. (b) Correspondıng $Y_{\mathrm{E}}^{\prime \prime}$ vs $Y_{\mathrm{E}}^{\prime}$ plots at the potential indicated

were performed at a less well polished polycrystalline electrode in p.a. $1 M \mathrm{HClO}_{4}$. The $Y_{\mathrm{E}}^{\prime \prime}$ vs. $Y_{\mathrm{E}}^{\prime}$ plot again yielded a straight line but with a phase angle increased to $4^{\circ}$. The rate of $\mathrm{H}^{+}$reduction is considerably larger at this electrode, as follows from the higher values of $R_{\mathrm{ct}}^{-1}$.

In order to examine the dispersion along the loci in Fig. $6 \mathrm{~b}$, the values of $Q^{-1}$ were determined by plotting $\log Y_{\mathrm{E}}^{\prime \prime}$ vs. $\log \omega$ (see eqn. 36). Next, the sets of $Q^{-1}, \alpha$ and $R_{\mathrm{ct}}$ values were used to re-calculate $Y_{\mathrm{E}}^{\prime}$ and $Y_{\mathrm{E}}^{\prime \prime} / \omega$ as functions of $\omega$. The agreement with the experimental values is very good, as can be seen in the typical example shown in Fig. 7. It is quite clear from this picture that the contribution of the CPE to the real component $Y_{\mathrm{E}}^{\prime}$ is by no means negligible with respect to $R_{\mathrm{ct}}^{-1}$,

TABLE 1

Rotation parameter $\alpha\left(\times 10^{2}\right)$ in perchlorate solutions

\begin{tabular}{llllll}
\hline$E / \mathrm{V}$ & $10^{2} \alpha$ & & & \\
\cline { 2 - 6 } & $\mathrm{Au}(111)$ & $\mathrm{Au}(100)$ & $\mathrm{Au}(110)$ & Poly Au & Poly Au \\
& $1 M \mathrm{HClO}_{4}$ & $1 M \mathrm{HClO}_{4}$ & $1 M \mathrm{HClO}_{4}$ & $1 M \mathrm{HClO}_{4}$ & $1 M \mathrm{NaClO}_{4}+$ \\
& & & & $636 \times 10^{-3} M \mathrm{HClO}_{4}$ \\
\hline-0.2 & $049^{a}$ & $100^{a}$ & $088^{a}$ & $1.39^{a}$ & $305^{a}$ \\
-0.3 & 0.29 & 144 & 1.07 & 126 & $270^{a}$ \\
-04 & 025 & 0.81 & 078 & 113 & $1.76^{a}$ \\
-05 & -006 & 033 & 025 & & 154 \\
-0.6 & & & & & 172 \\
-0.7 & & & & & 176 \\
\hline
\end{tabular}

${ }^{a}$ Measured outside the faradaic region. 
despite the quite low value of $\alpha$. Also, a considerable frequency dispersion is found in the "apparent double-layer capacity" $Y_{\mathrm{E}}^{\prime \prime} / \omega$.

Finally, in Fig. 8 the obtained values of $R_{\mathrm{ct}}^{-1}$ and $Q^{-1}$ are represented as functions of $\mathrm{dc}$ potential for the polycrystalline electrode. The $R_{\mathrm{ct}}^{-1}$ data were analysed following the procedures described in Section (III) and combined with results from dc current responses to potential step perturbations as described in the previous paper [20]. With the resulting $k_{\mathrm{f}}(E)$ values, a theoretical $R_{\mathrm{ct}}^{-1}$ vs. $E$ curve was calculated in order to demonstrate the precision of agreement with the experimental points. In the $Q^{-1}$ vs. $E$ plot the corresponding values of $Y_{\mathrm{E}}^{\prime \prime} / \omega$ at $220 \mathrm{~Hz}$ and of $C_{0}$, cf. eqn. (5), are also given, thus indicating the typical differences between the three quantities.

Similar results as shown in Figs. 6-8 were obtained in the experiments with the single crystalline electrodes and with the polycrystalline electrode in $1 \mathrm{M} \mathrm{HClO}_{4}$. The results of these experiments in terms of the potential dependence of $\ln k_{\mathrm{f}}(E)$ have been discussed in the previous paper [20]. Here we only note that evidently the proton reduction at gold electrodes leads to an ac behaviour concordant with the equivalent circuits of Fig. 4. It may be interesting to refer to the early work of Gerischer and Mehl [21], who derived theoretical expressions for the faradaic admittance in the case where non-stationary adsorption of intermediate atomic hydrogen plays a significant role in the reaction mechanism. The frequency dependence of this admittance obeys the relationship

$Y_{\mathrm{F}}=R^{-1}+R_{\mathrm{O}}^{-1} \frac{G^{2}+\imath \omega C_{\mathrm{H}} G}{G^{2}+\omega^{2} C_{\mathrm{H}}^{2}}$

where we have used a notation somewhat different from that of Gerischer and Mehl.

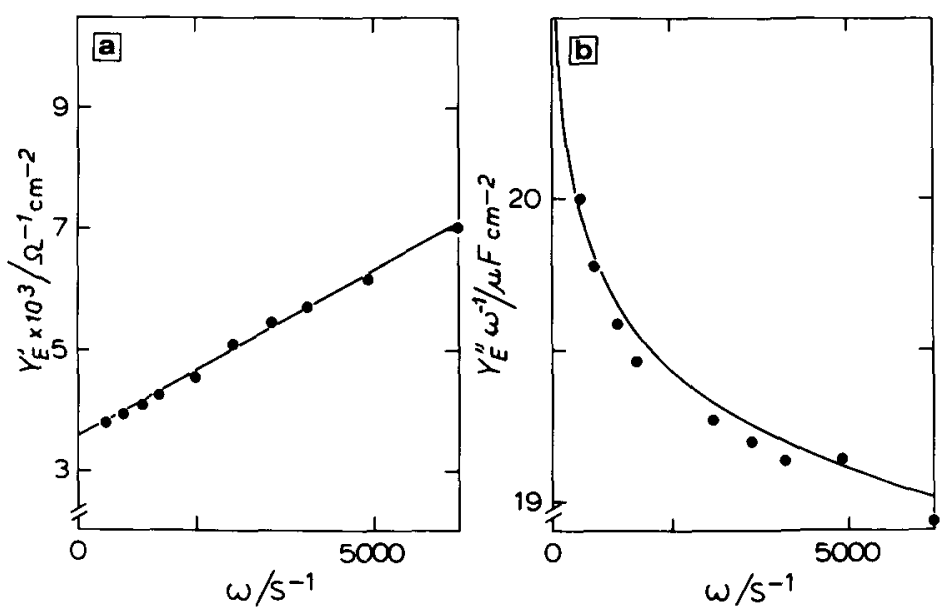

Fig 7. (a) $Y_{\mathrm{E}}^{\prime}$ vs $\omega$, (b) $Y_{\mathrm{E}}^{\prime \prime} / \omega$ vs $\omega$ for $\mathrm{H}^{+}$reduction in $6.36 \times 10^{-3} M \mathrm{HClO}_{4}+1 M \mathrm{NaClO}_{4}$, at $E=-0.69 \mathrm{~V}$ vs SSCE. (๑) Measured points, (- $(-)$ calculated curves (see text) 
The meaning of the symbols is given in ref. 20 . If the faradaic admittance of eqn. (39) is supposed to be in parallel with a double-layer admittance $Y_{\mathrm{c}}=\imath \omega C_{\mathrm{d}}$, it is possible to predict the corresponding $Y_{\mathrm{E}}^{\prime \prime}$ vs. $Y_{\mathrm{E}}^{\prime}$ diagram. Gerischer and Mehl tried to verify their theory at copper and silver electrodes and in fact observed a frequency dispersion in $Y_{\mathrm{E}}^{\prime}$, which, however, was said to be "spurious" rather than a confirmation of the theory. It seems that this conclusion was quite correct and that the observed effects were due to the presence of a CPE.

\section{(V.2) The reduction of tris-oxalato $\mathrm{Fe}(I I I)$}

This system is of interest for the present treatment because it can be expected to be quasi-irreversible and therefore the frequency dependence of $Y_{\mathrm{E}}^{\prime}$ and $Y_{\mathrm{E}}^{\prime \prime}$ may originate from the faradaic contribution as well as from the CPE. The standard heterogeneous rate constant of the reaction at a gold electrode has been reported by Randles and Somerton [22] to have the value $k_{\text {sh }}=5 \times 10^{-3} \mathrm{~cm} \mathrm{~s}^{-1}$ (at $E^{0}=-0.231$ $\mathrm{V}$ vs. SCE). We intend to present a study of the reaction rate as a function of potential in a later publication [23], and here confine the discussion to the analysis problem.

In Fig 9a, a complex plane diagram of the interfacial admittance at three different potentials is represented. At first sight the plots are not very dissimilar to those in Fig. 6b, 1.e. almost straight lines are obtained, with only slight bending at the lower frequencies. It is therefore tempting to consider the high-frequency part as obeying ac irreversible behaviour and consequently to determine $\alpha$ and $R_{\mathrm{ct}}^{-1}$ by extrapolation of this part to $\omega=0$ in the same way as we did for the $\mathrm{H}^{+} / \mathrm{H}_{2}$ reaction. Next, we can try to interpret the low frequency data by means of eqn. (37), so that at the end all the system parameters, $\alpha, Q^{-1}, R_{\mathrm{ct}}$ and $p^{\prime}$ or $R_{\mathrm{ct}} / p^{\prime}=\sigma$, should be known.

The results of such a procedure, however, appeared unsatısfactory, especially at potentials close to the polarographic half-wave potential. The values of $R_{\mathrm{ct}}$ and $p^{\prime}$ did not yield the same value for the rate constant, or, in other words, the Warburg
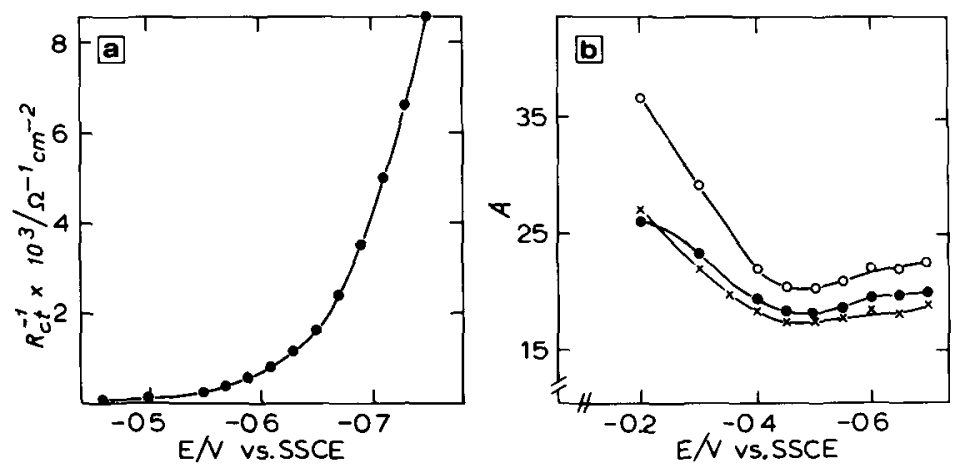

Fig. 8 (a) $R_{\mathrm{ct}}^{-1}$ vs potential for $636 \times 10^{-3} M \mathrm{HClO}_{4}$ in $1 M \mathrm{NaClO}_{4}$ (b) "Capacitance" vs potential $A(E)$ corresponds respectively to $Y_{\mathrm{E}}^{\prime \prime} / \omega$ at $220 \mathrm{~Hz}(\bullet), Q^{-1}(O)$ and $C_{0}(\times)$ 


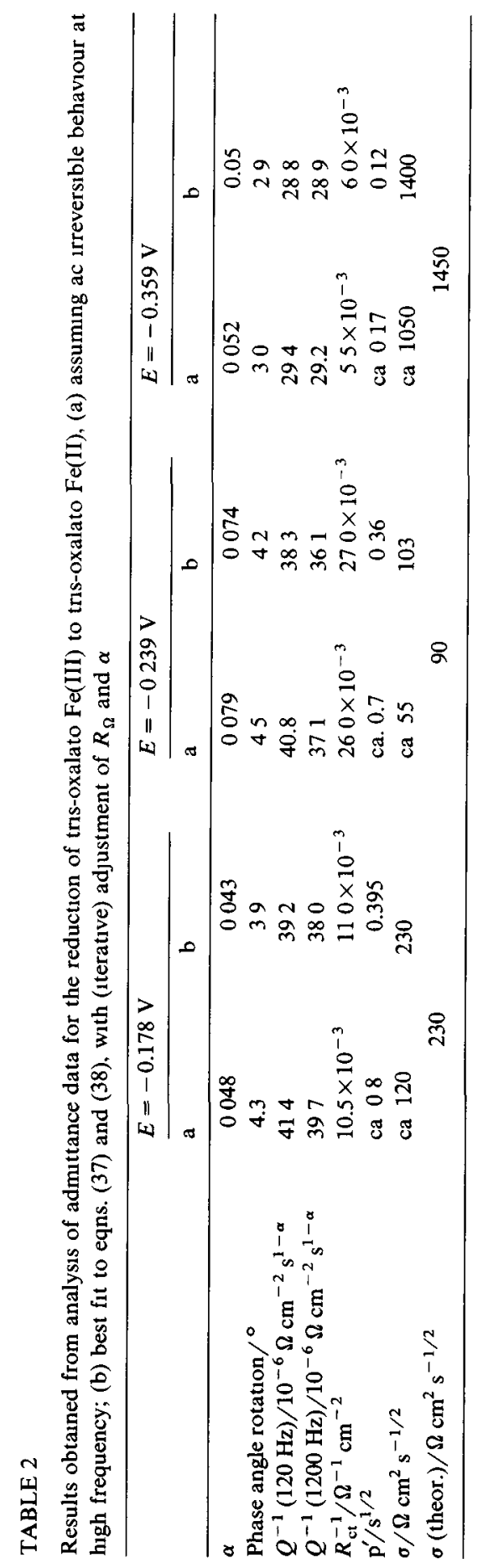


coefficient $\sigma$ was not found to agree with its theoretical value. In addition, $Q^{-1}$ values were not independent of frequency. The conclusion is that also at the higher frequencies, the factor containing $p$ in eqn. (37) is different from unity (the irreversible limit). The apparent linearity of the $Y_{E}^{\prime \prime}$ vs. $Y_{E}^{\prime}$ plot is due to the fact that for large $p^{\prime}$, both $Y_{\mathrm{F}}^{\prime}$ and $Y_{\mathrm{F}}^{\prime \prime}$ approach (almost) linear functions of $\omega^{-1 / 2}$, according to

$$
\begin{array}{ll}
\lim _{p \rightarrow \infty} Y_{\mathrm{F}}^{\prime}=R_{\mathrm{ct}}^{-1}\left[1-\frac{1}{p}\right] & (p>3) \\
\lim _{p \rightarrow \infty} Y_{\mathrm{F}}^{\prime \prime}=R_{\mathrm{tt}}^{-1} \frac{1}{p+2} \quad(p>5)
\end{array}
$$

The corresponding approximation of eqn. (38) is

$$
\lim _{p \rightarrow \infty} Y_{\mathrm{E}}^{\prime}-Y_{\mathrm{E}}^{\prime \prime} \operatorname{tg}(\alpha \pi / 2)=R_{\mathrm{ct}}^{-1}\left[1-\frac{1}{p}-\frac{\operatorname{tg}(\alpha \pi / 2)}{p+2}\right] \quad(p>3)
$$

In Fig. $9 \mathrm{~b}$ it can be seen that in our case the best fit of the rigorous eqn. (38) to our data (i.e. the drawn lines) is indeed found to have a linear dependence on $\omega^{-1 / 2}$. The intercepts at $\omega^{-1 / 2}=0$ give $R_{\mathrm{ct}}^{-1}$ and the slopes are equal to $R_{c t}^{-1} / p^{\prime}$. The data obtained are satisfactorily consistent, as can be deduced from Table 2, where the results from the two procedures are compared. The re-calculated $Y_{\mathrm{E}}^{\prime \prime}$ vs. $Y_{\mathrm{E}}^{\prime}$ plots are shown in Fig. 9a as the drawn curves. In addition, the dashed straight lines have been drawn to show the real ac irreversible limits, 1.e. straight lines intersectıng the $Y_{E}^{\prime}$ axis at $Y_{E}^{\prime}=R_{c t}^{-1}$, and having a slope equal to $\cot (\alpha \pi / 2)$.
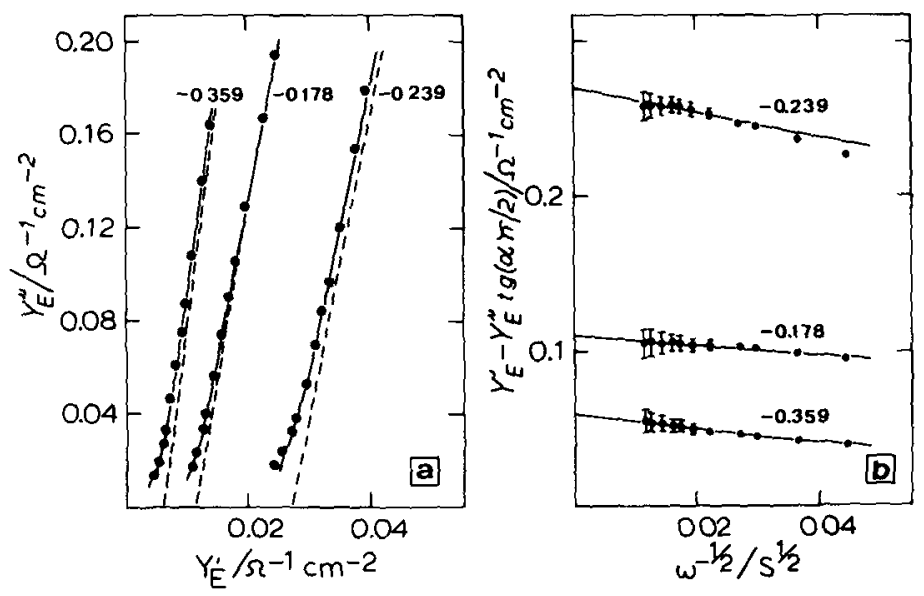

Fig. 9 (a) $Y_{\mathrm{E}}^{\prime \prime}$ vs. $Y_{\mathrm{E}}^{\prime}$ plots at the indicated potentials for the reduction of tris-oxalato $\mathrm{Fe}(\mathrm{III})(\bullet)$ Measured points, ( $(-)$ theoretical curves. (.....) limitıng dc irreversible behaviour. (b) Plots of $Y_{\mathrm{E}}^{\prime}-Y_{\mathrm{E}}^{\prime \prime} \operatorname{tg}(\alpha \pi / 2)$ vs. $\omega^{-1 / 2}$. (๑) Measured points; $(-)$ theoretical curves. The vertical bars represent deviations corresponding to $\Delta \alpha=0.005$ (see Text). 
It should be emphasized that the proposed analysis is preceded by the adjustment of two system parameters, namely $R_{\Omega}$ and $\alpha$, whereas in the classical case, where $\alpha=0$, only $R_{\Omega}$ has to be chosen. A misestimation of $\alpha$ will have an effect on the quantity $Y_{\mathrm{E}}^{\prime}-Y_{\mathrm{E}}^{\prime \prime} \operatorname{tg}(\alpha \pi / 2)$ similar to a misestimation of $R_{\Omega}$ : an error is introduced which increases with increasing frequency. We have indicated this in Fig. $9 \mathrm{~b}$ by means of vertical bars through the experimental points. These bars correspond to a variation in $\alpha$ of \pm 0.003 [i.e. $\pm 0.005 \mathrm{in} \operatorname{tg}(\alpha \pi / 2)$ ]. A variation in $R_{\Omega}$ of $\pm 0.03 \Omega \mathrm{cm}^{2}$ would produce an equal error at the highest frequency (here only $1200 \mathrm{~Hz}$ !), with a stronger tendency to decrease at the lower frequencies. The conclusion is that after a misestimation of $R_{\Omega}$, most inevitably $\alpha$ also will be adjusted to an erroneous value in order to obtain a reasonable frequency dependence of $Y_{\mathrm{E}}^{\prime}-Y_{\mathrm{E}}^{\prime \prime} \operatorname{tg}(\alpha \pi / 2)$. On the other hand, this kind of "compensating" adjustment helps us to find a reliable value for $R_{\mathrm{ct}}^{-1}$ which can be employed as a starting value for further fitting and eventual re-adjustment of $R_{\Omega}$ and $\alpha$.

\section{(VI) CONCLUSION}

The most important result of the present work is, in our opinion, that it is shown how severely the CPE influences the frequency dispersion of the interfacial admittance even in the case of quite small rotation angles $\left(2-4^{\circ}\right)$. Especially at such small angles the effect is likely to be ignored, which would lead to large errors in the determination of faradaic rate parameters and thus to serious misinterpretations. However, it is quite feasible to account for the CPE effect correctly and to check the results of the analysis on their internal consistency.

In the literature up to now, the analysis of electrode impedances in the case where the double-layer behaves as a constant phase element was made on the tacit assumption that the CPE simply replaces the double-layer capacity in any equivalent circuit without having a relation with the other constituents, which is logical if the CPE behaviour is a property of the double-layer itself and not due to the distribution of $R_{\Omega} C_{\mathrm{d}}$ values originating from surface inhomogeneity. Curiously enough, the latter explanation is usually adopted and the former only rather rarely mentioned as a possibility with hardly any physical justıfication.

Consequently, the analyses reported in the literature are in fact internally conflicting procedures, combining a distributed relaxation time for double-layer charging with a modified Randles's circuit. In addition, reported values of the double-layer capacity, obtained by " $C_{\mathrm{d}} "=Y_{\mathrm{E}}^{\prime \prime} / \omega$ at some frequency, are in fact values of an irrational quantity in the case where CPE behaviour occurs. A more rational double-layer parameter appears to be the quantity $C_{0}$, but the essentral meaning of this quantity is also obscure as long as the proper fundamental background of the CPE remains unclarified.

From the methodological considerations presented in the theoretical part of this paper, it can be concluded that the analysis of an electrode impedance comprising both a faradaic impedance and a CPE may offer a possibility by experıment to discriminate between the two possible explanations. To this end, it is required that 
the faradaic admittance should have a substantial magnitude as compared to the reciprocal ohmic resistance (explicitly the factor $\left(1+R_{\Omega} Y_{\mathrm{F}}\right)^{\alpha}$ should differ significantly from unity). Unfortunately, the circumstances of the experiments discussed here did not meet this requirement, mostly because of the irreversible nature of the two systems studied. We intend to search for a more suitable system to investigate this particular aspect in the near future.

\section{REFERENCES}

1 R. de Levie, J Electroanal Chem., 9 (1965) 117

2 D.C Grahame, J Am Chem Soc., 68 (1946) 301

3 J Newman, J. Electrochem. Soc , 117 (1970) 198

4 R. de Levie, Electrochum Acta, 10 (1965) 113

5 S. Isekı, K. Ohashı and S Nagura, Electrochım. Acta, 17 (1972) 2249

6 R D. Armstrong and W I Archer, J. Electroanal. Chem., 87 (1978) 221

7 W Scheider, J Phys Chem., 79 (1975) 127 and references cited therein

8 P.H Bottelberghs and G.H.J Broers, J. Electroanal Chem., 67 (1976) 155.

9 H Fricke, Philos. Mag., 14 (1932) 310

$10 \mathrm{R}$. de Levie in P Delahay and $\mathrm{W}$ Tobias (Eds.), Advances in Electrochemistry and Electrochemical Engineenng, Vol. 6, Interscience, New York, 1967, p 329.

11 J.E B. Randles, Discuss. Faraday Soc., 1 (1947) 11

12 N G. van Kampen, Ned T Natuurkunde, 24 (1958) 1.

13 K.S Cole and R.H. Cole, J. Chem Phys, 9 (1941) 341

14 R.M. Fuoss and J G Kırkwood, J. Am. Chem. Soc., 63 (1941) 385

15 K S. Cole and R.H. Cole, J. Chem. Phys, 10 (1942) 98.

16 A Hamelın, T Vitanov, E. Sevestyanov and A. Popov, J. Electroanal Chem 145 (1983) 225

17 A J. Bard and L.R Faulkner, Electrochemical Methods, Wiley, New York, 1980, p 166

18 M. Sluyters-Rehbach and J.H Sluyters in J O'M Bockris and E Yeager (Eds), Comprehensive Treatıse of Electrochemistry, Vol. 9, Plenum Press, New York, in press

19 J.H Sluyters, Rec Trav Chım, Pays-Bas, 79 (1960) 1092.

20 G J Brug, M Sluyters-Rehbach, J.H Sluyters and A Hamehn, J Electroanal. Chem., in press

$21 \mathrm{H}$ Genscher and W. Mehl, Z. Elektrochem., 59 (1959) 1049.

22 J E B. Randles and K.W. Somerton, Trans. Faraday Soc, 48 (1952) 937

23 G.J Brug, M Sluyters-Rehbach and J H Sluyters, J. Electroanal Chem, 176 (1984) 297. 